\title{
Power Allocation for 5G Mobile Multiuser Cooperative Networks
}

\author{
Fagen Yin $\mathbb{D}^{1}$ and Wencai Du $\mathbb{D}^{2}$ \\ ${ }^{1}$ College of Physical Science and Engineering, Yichun University, Yichun 336000, Jiangxi, China \\ ${ }^{2}$ The Institute of Data Engineering and Sciences, University of Saint Joseph, Macao 999078, China \\ Correspondence should be addressed to Fagen Yin; ynx522@163.com and Wencai Du; george.du@usj.edu.mo
}

Received 15 November 2021; Revised 6 December 2021; Accepted 15 December 2021; Published 28 December 2021

Academic Editor: Sang-Bing Tsai

Copyright (c) 2021 Fagen Yin and Wencai Du. This is an open access article distributed under the Creative Commons Attribution License, which permits unrestricted use, distribution, and reproduction in any medium, provided the original work is properly cited.

\begin{abstract}
With the fifth generation $(5 \mathrm{G})$ communication technology, the mobile multiuser networks have developed rapidly. In this paper, the performance analysis of mobile multiuser networks which utilize decode-and-forward (DF) relaying is considered. We derive novel outage probability (OP) expressions. To improve the OP performance, we study the power allocation optimization problem. To solve the optimization problem, we propose an intelligent power allocation optimization algorithm based on grey wolf optimization (GWO). We compare the proposed GWO approach with three existing algorithms. The experimental results reveal that the proposed GWO algorithm can achieve a smaller OP, thus improving system efficiency. Also, compared with other channel models, the OP values of the 2 -Rayleigh model are increased by $81.2 \%$ and $66.6 \%$, respectively.
\end{abstract}

\section{Introduction}

Recently, the increasing provision of multiuser services, the ever-increasing number of devices, and the continuous growth of data pose significant challenges to massive mobile multiuser connectivity. Fifth generation (5G) mobile communication networks are very important in achieving massive mobile multiuser connectivity $[1,2]$. To meet this requirement, the boom of $5 \mathrm{G}$ mobile communications has resulted in the emergence of many new technologies [3-5]. Nonorthogonal multiple access and millimeter-wave communications are key aspects of 5G technology [6]. However, the complex multiuser communication environment makes the $5 \mathrm{G}$ mobile communication challenging.

As an alternative way to ensure reliable multiuser communication, cooperative communication has sparked a great deal of research [7]. Secrecy performance of multiplerelay cooperative communication was investigated in [8]. In [9], cooperative cognitive relaying was employed to provide secure communications. Xu et al. [10] studied the incremental decode-and-forward (DF) cooperative relay network.

To improve the multiuser cooperative communication, power allocation plays a key role [11]. Xu et al. employed the passive beamforming to improve energy efficiency optimization in [12]. In [13], with multicarrier division, Li et al. investigated resource allocation problem. Filomeno et al. proposed two power allocation algorithms in [14].

To further improve the power allocation performance, various swarm intelligence optimization methods have been used to optimize the parameters [15]. To solve the multiUAV task allocation problem, an improved genetic algorithm (GA) was proposed in [16]. An adaptive firefly algorithm (FA) algorithm was proposed to enhance data security in [17]. By using the golden section (GS) algorithm, Cuevas et al. optimized the evolutionary computation in [18].

However, research on power allocation optimization of mobile multiuser communications is very rare. Therefore, we investigate power allocation optimization over the 2-Rayleigh model. The main contributions are as follows:

(1) With transmit antenna selection (TAS), we analyze the OP performance of mobile multiuser networks. New OP expressions are derived. These results are more complex than those in the Rayleigh model.

(2) To improve the OP performance, we propose an intelligent power allocation optimization method 
based on grey wolf optimization (GWO), which reduces computational complexity.

(3) Compared with Nakagami and Rayleigh channel models, the 2-Rayleigh model has an increase of $81.2 \%$ and $66.6 \%$ in OP values, respectively. We also test the firefly algorithm (FA), the genetic algorithm (GA), and the golden section (GS) algorithm. Compared with these algorithms, our proposed GWO method achieves a smaller OP.

Table 1 shows the notations in our paper.

\section{System Model}

In Figure 1, $N_{t}$ and $N_{r}$ antennas are installed at mobile source (MS) and mobile relay (MR), respectively. There are $L$ mobile users (MUs). The channel coefficient $h$ follows 2-Rayleigh distribution [19]. The energy $E$ is allocated by $K$. $W_{\left\{\mathrm{SU}_{i}, \mathrm{RUj}_{j}\right\}}$ are the position gains of $\mathrm{MS}_{i} \longrightarrow \mathrm{MU}_{l}$ and $\mathrm{MR}_{j} \longrightarrow \mathrm{MU}_{l}$, respectively.

Firstly, $\mathrm{MU}_{l}$ and $\mathrm{MR}_{j}$ receive the signals as

$$
\begin{aligned}
& r_{\text {SUil }}=\sqrt{W_{\text {SUil }} K E} h_{\text {SUil }} x+N_{\text {SUil }}, \\
& r_{S R i j}=\sqrt{K E} h_{S R i j} x+N_{S R i j},
\end{aligned}
$$

where $N_{\mathrm{SR} i j}$ and $N_{\mathrm{SU} i l}$ are Gaussian noises.

Then, $\mathrm{MR}_{j}$ employs DF scheme and transmits signal to $\mathrm{MU}_{l}$ as

$$
r_{R U j l}=\beta \sqrt{W_{R U j l}(1-K) E} h_{R U j l} x+N_{R U j l} .
$$

The SNR $\gamma_{\mathrm{SR} i j}$ at $\mathrm{MR}_{j}$ is given as

$$
\begin{aligned}
\gamma_{S R i j} & =\frac{K\left|h_{S R i j}\right|^{2} E}{N_{0}} \\
& =K\left|h_{S R i j}\right|^{2} \bar{\gamma} .
\end{aligned}
$$

If $\gamma_{\mathrm{Sr} i}<\gamma_{\mathrm{th}}, \mathrm{MU}_{l}$ cannot receive the signal from MR. $\gamma_{\mathrm{Sr} i}$ is given as
TABLE 1: Notations.

\begin{tabular}{lc}
\hline Notations & Designation \\
\hline$K$ & Power allocation coefficient \\
$W$ & The position gain \\
SNR & Signal-to-noise ratio \\
$N_{t}$ & The transmit antennas \\
$N_{r}$ & The receive antennas \\
\hline & \\
& $\gamma_{S R i}=\max _{1 \leq j \leq N_{r}}\left(\gamma_{S R i j}\right)$.
\end{tabular}

$\mathrm{MU}_{l}$ receives the SNR $\gamma_{i l}$ aswhere

$$
\begin{aligned}
& \gamma_{\text {SUil }}=K W_{\text {SUil }}\left|h_{\text {SUil }}\right|^{2} \bar{\gamma}, \\
& \gamma_{\text {RUil }}=(1-K) W_{\text {RUil }}\left|h_{\text {RUil }}\right|^{2} \bar{\gamma},
\end{aligned}
$$

where $\bar{\gamma}$ is the average SNR.

The best user is chosen from $L$ mobile users:

$$
\gamma_{i}=\max _{1 \leq l \leq L}\left(\gamma_{i l}\right)
$$

The TAS is employed to select $w$ as

$$
\begin{aligned}
w & =\max _{1 \leq i \leq N_{t}}\left(\gamma_{i}\right) \\
& = \begin{cases}\max _{1 \leq i \leq N_{t}, 1 \leq l \leq L}\left(\gamma_{S U i l}\right), & \text { if }|C|=0, \\
\max _{1 \leq i \leq N_{t}, 1 \leq l \leq L, j \in C}\left(\gamma_{S U i l}, \gamma_{R U j l}\right), & \text { if }|C| \neq 0,\end{cases}
\end{aligned}
$$

where $C$ is given as

$$
C=\left\{1 \leq i \leq N_{t} \mid \gamma_{S R i} \geq \gamma_{t h}\right\}
$$

\section{OP Performance with TAS}

We obtains the OP as

$$
F=Q_{1}+Q_{2},
$$

where

$$
\begin{aligned}
Q_{1} & =\left(G_{1,3}^{2,1}\left[\frac{\gamma_{t h}}{K \bar{\gamma}_{1,1,0}^{1}}\right]\right)^{N_{t} \times N_{r}} \times\left(G _ { 1 , 3 } ^ { 2 , 1 } \left[\frac{R_{t h}}{\left.\left.K W_{S U} \bar{\gamma}_{1,1,0}^{1}\right]\right)^{N_{t} \times L}},\right.\right. \\
Q_{2} & =\sum_{n=1}^{N_{t}}\left(\begin{array}{c}
N_{t} \\
n
\end{array}\right)\left(G_{1,3}^{2,1}\left[\frac{\gamma_{t h}}{K \bar{\gamma}_{1,1,0}^{1}}\right]\right)^{\left(N_{t}-n\right) \times N_{r}}\left(1-\left(G_{1,3}^{2,1}\left[\frac{\gamma_{t h}}{K \bar{\gamma}_{1,1,0}^{1}}\right]\right)^{N_{r}}\right)^{n} \\
& \times\left(G_{1,3}^{2,1}\left[\frac{R_{t h}}{K W_{S U} \bar{\gamma}} \mid 1,1,0_{1}\right]\right)^{N_{t} \times L}\left(G_{1,3}^{2,1}\left[\frac{R_{t h}}{(1-K) W_{R U} \bar{\gamma}} \mid 1,1,0_{1}\right]\right)^{n \times L},
\end{aligned}
$$

where $R_{\mathrm{th}}$ is a given threshold. 


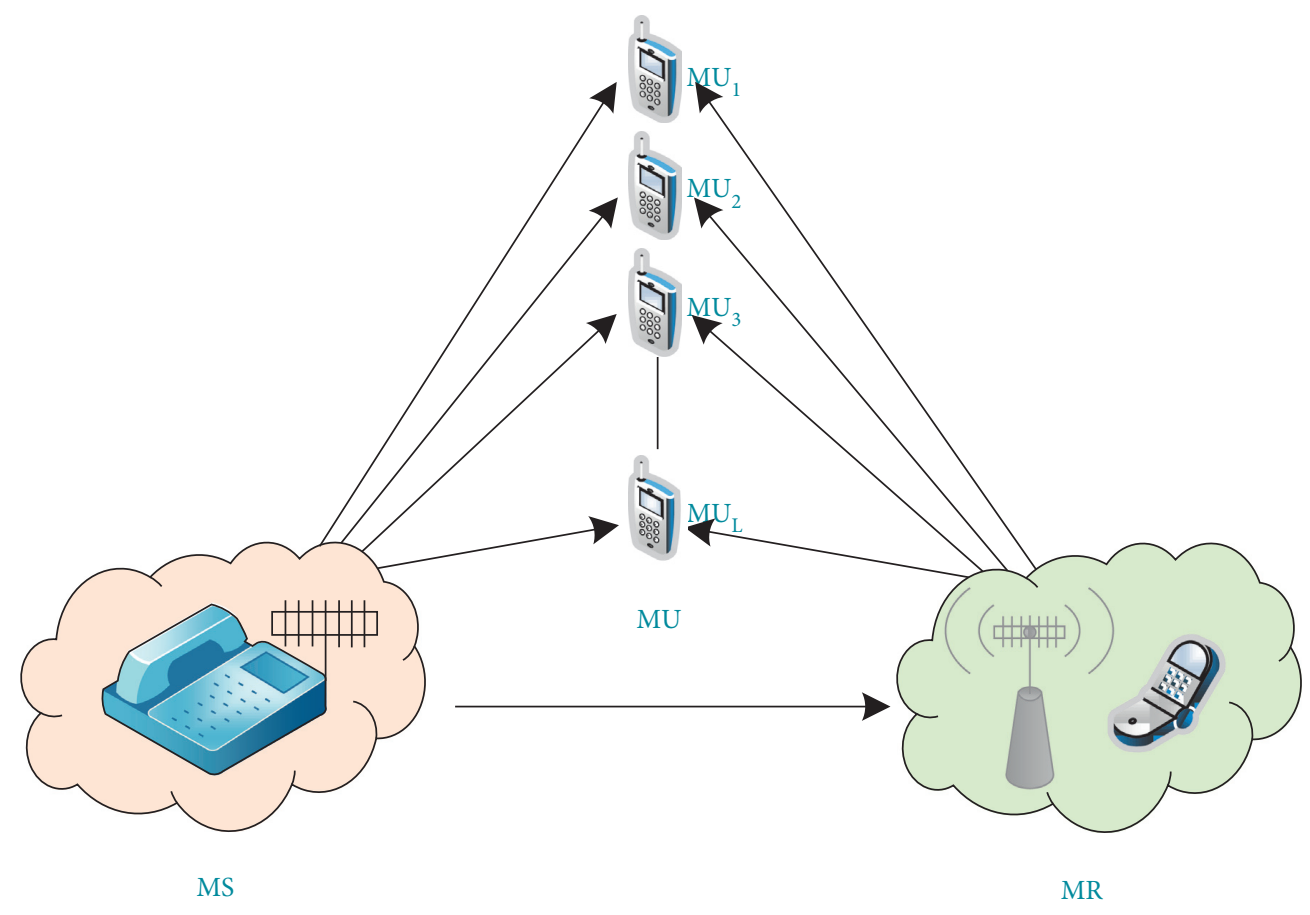

Figure 1: The system model.

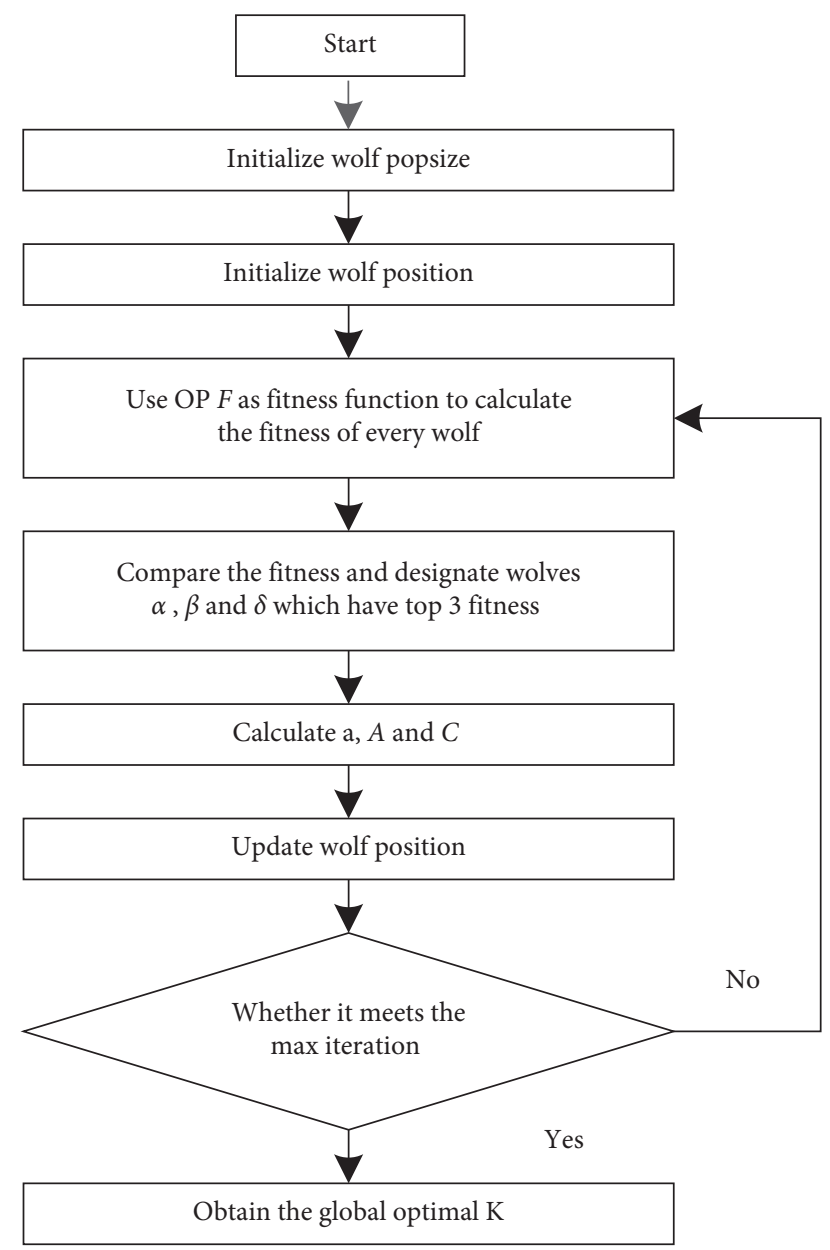

Figure 2: GWO algorithm. 


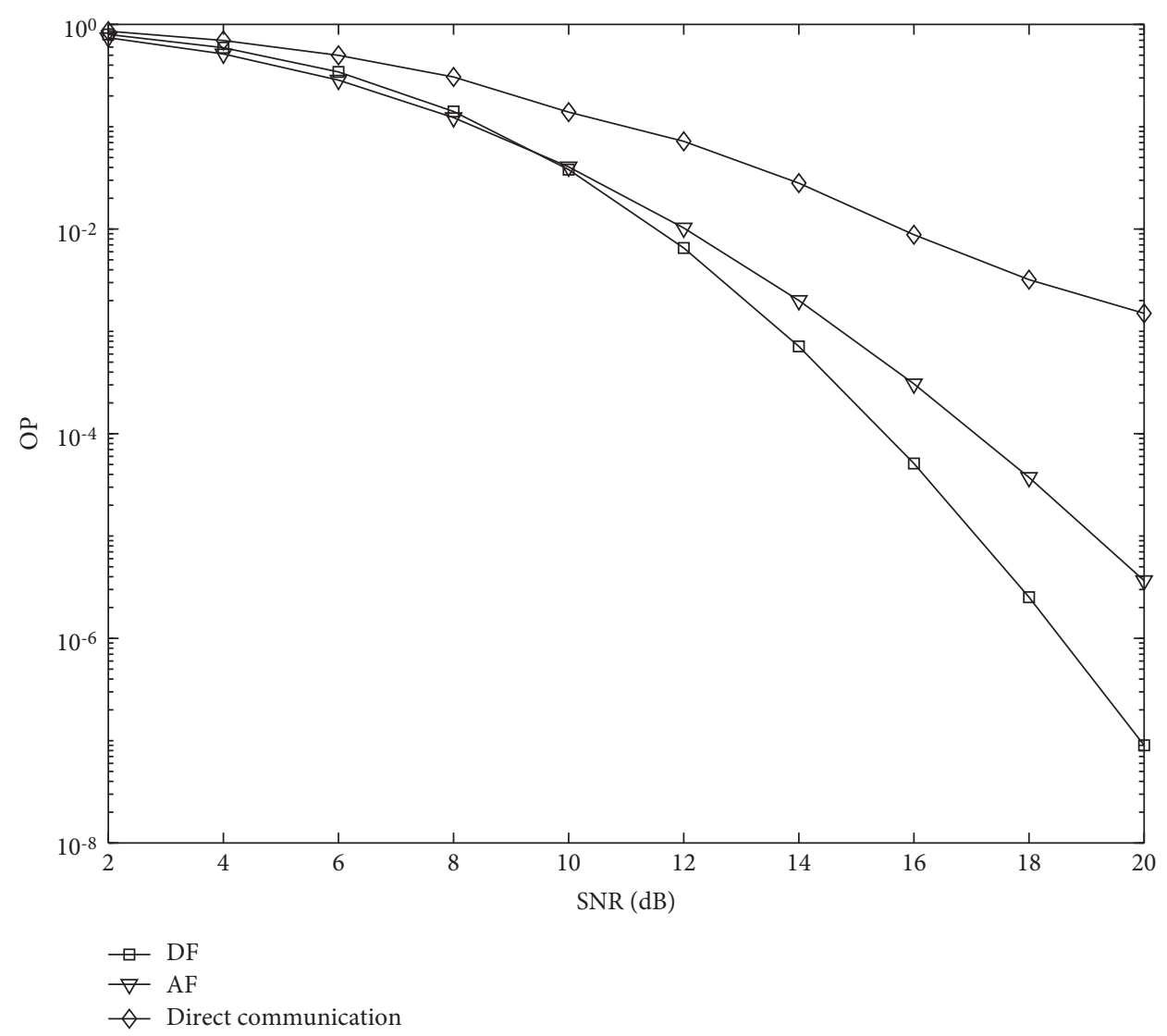

Figure 3: OP comparison with different schemes.

\section{Power Allocation Intelligent Optimization}

According to [20-22], the GWO algorithm is divided into the following parts.

4.1. Encircling. The encircling process is expressed as

$$
\begin{aligned}
D D & =\left|C \cdot X X_{p}(t)-X X(t)\right|, \\
X X(t+1) & =X X_{p}(t)-A \cdot D D, \\
A & =2 a \cdot r_{1}-r_{2}, \\
C & =2 \cdot r_{2},
\end{aligned}
$$

where $r_{1}, r_{2} \in[0,1]$ and $a \in[0,2]$.

4.2. Hunting. The wolves renew their positions as

$$
X X(t+1)=\frac{X X_{1}+X X_{2}+X X_{3}}{3},
$$

where

$$
\begin{aligned}
& X X_{1}=X X_{\alpha}(t)-A_{\alpha} \cdot D D_{\alpha}, \\
& X X_{2}=X X_{\beta}(t)-A_{\beta} \cdot D D_{\beta}, \\
& X X_{3}=X X_{\delta}(t)-A_{\delta} \cdot D D_{\delta}, \\
& D D_{\alpha}=\left|C_{\alpha} \cdot X X_{\alpha}(t)-X X(t)\right|, \\
& D D_{\beta}=\left|C_{\beta} \cdot X X_{\beta}(t)-X X(t)\right|, \\
& D D_{\delta}=\left|C_{\delta} \cdot X X_{\delta}(t)-X X(t)\right| .
\end{aligned}
$$

4.3. Attacking. The wolves attack the prey. The maximum iteration is ter. $a$ is given as

$$
a=2-\frac{2 t}{\text { ter }}
$$

Figure 2 shows the GWO algorithm.

\section{Performance Results}

Figure 3 illustrates the comparison of amplify-and-forward (AF), DF, and direct communication schemes. Table 2 shows 
TABle 2: Parameters of Figure 3.

\begin{tabular}{ll}
\hline$\mu$ & $0 \mathrm{~dB}$ \\
$K$ & 0.5 \\
$\gamma_{\text {th }}$ & $5 \mathrm{~dB}$ \\
$R_{\text {th }}$ & $5 \mathrm{~dB}$ \\
$N_{\mathrm{t}}$ & 2 \\
$N_{\mathrm{r}}$ & 2 \\
$L$ & 2 \\
\hline
\end{tabular}

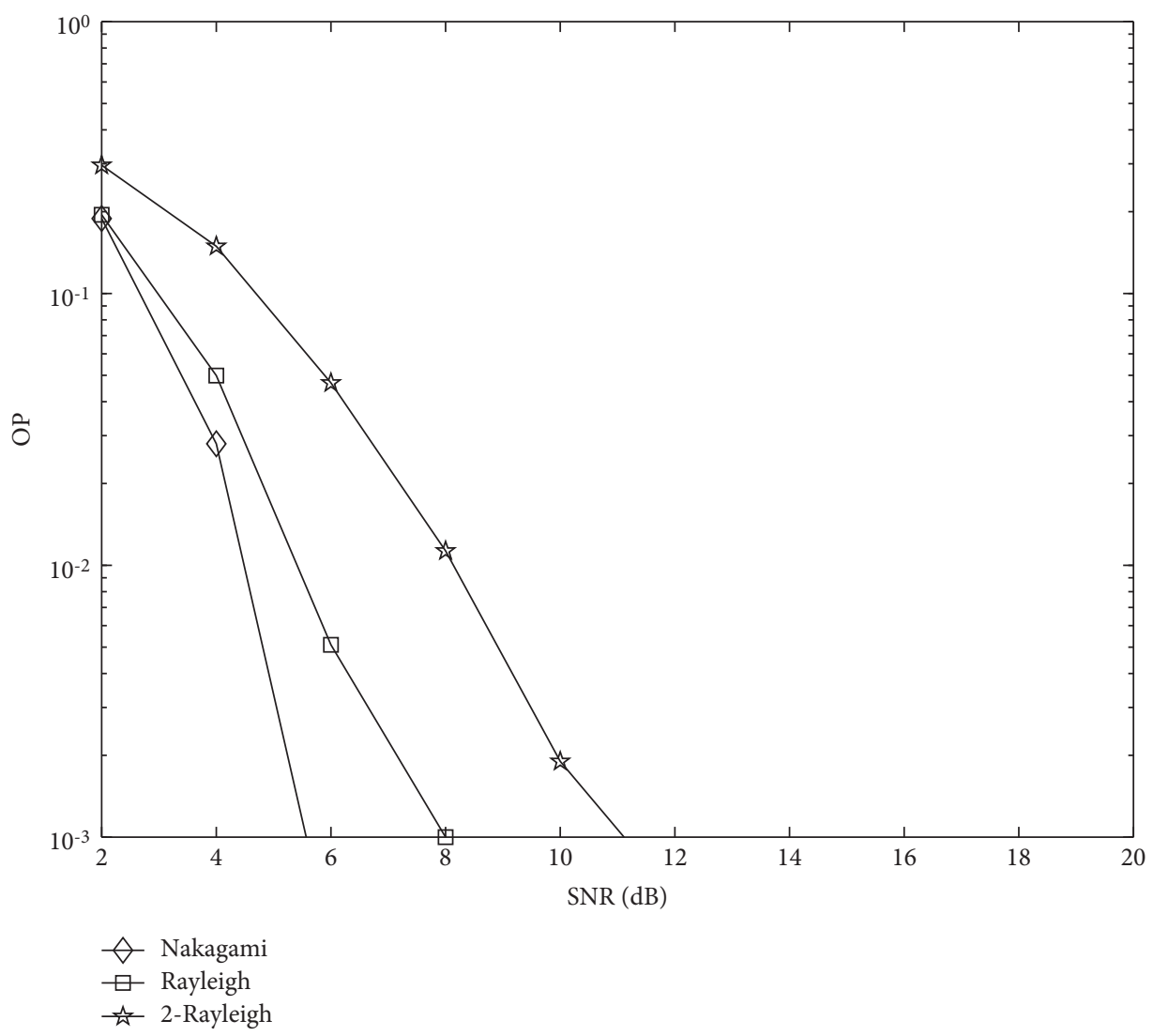

FIGURE 4: OP comparison with different channel models.

the corresponding parameters. The DF scheme is superior to $\mathrm{AF}$ and direct communication schemes. This means that with the increase of SNR, the cooperative communication condition becomes good, which reduces the OP. Compared with direct transmission, it also shows that cooperative transmission always reduces the OP.

Figure 4 presents the OP performance comparison under Nakagami, Rayleigh, and 2-Rayleigh models. The parameters are given in Table 3. We can see that the OP performance of the Nakagami model is better than that of Rayleigh and 2 -Rayleigh models. When $\mathrm{SNR}=4 \mathrm{~dB}$, the $\mathrm{OP}$ values are $0.0280,0.0499$, and 0.1492 , respectively. Compared with Nakagami and Rayleigh channel models, the 2-Rayleigh model has an increase of $81.2 \%$ and $66.6 \%$ in OP values, respectively.
Table 3: Parameters of Figure 4.

\begin{tabular}{ll}
\hline$\mu$ & $0 \mathrm{~dB}$ \\
$K$ & 0.6 \\
$\gamma_{\text {th }}$ & $5 \mathrm{~dB}$ \\
$R_{\text {th }}$ & $5 \mathrm{~dB}$ \\
$N_{t}$ & 2 \\
$N_{r}$ & 2 \\
$L$ & 2 \\
\hline
\end{tabular}

In Figures 5-8, we obtain the optimum $K$ for the GWO, GS, GA, and FA methods. The parameters are given in Table 4. Compared with GS, GA, and FA, GWO achieves a smaller OP (0.0005). This is due to the fact that GWO has a simple structure and a strong convergence performance, which is easy to implement. 


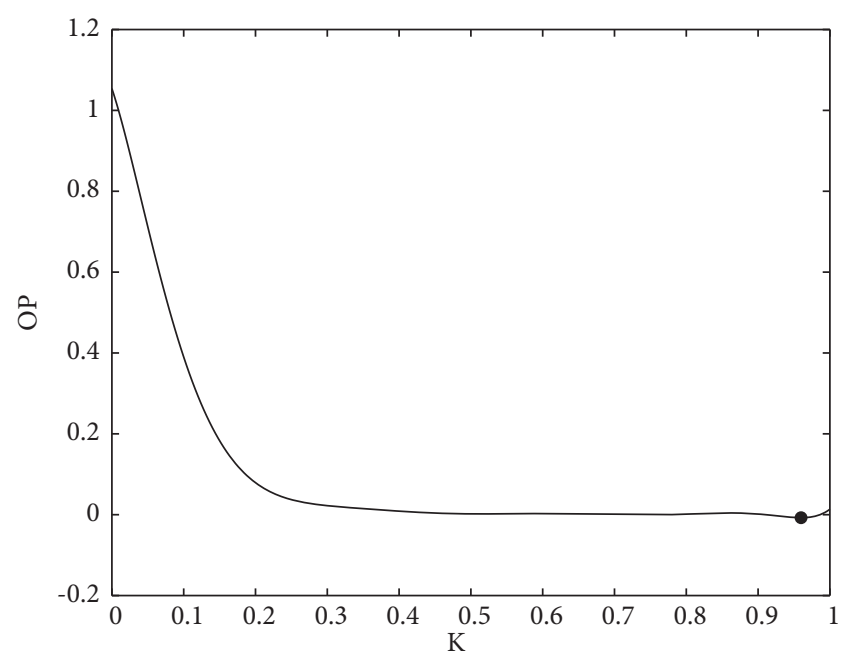

Figure 5: Optimum $K$ of GWO.

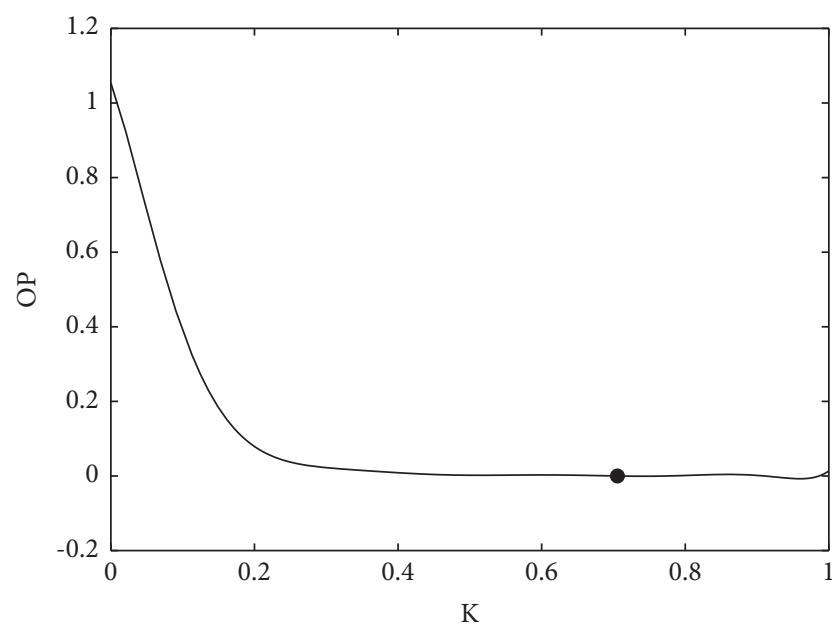

Figure 6: Optimum $K$ of GA.

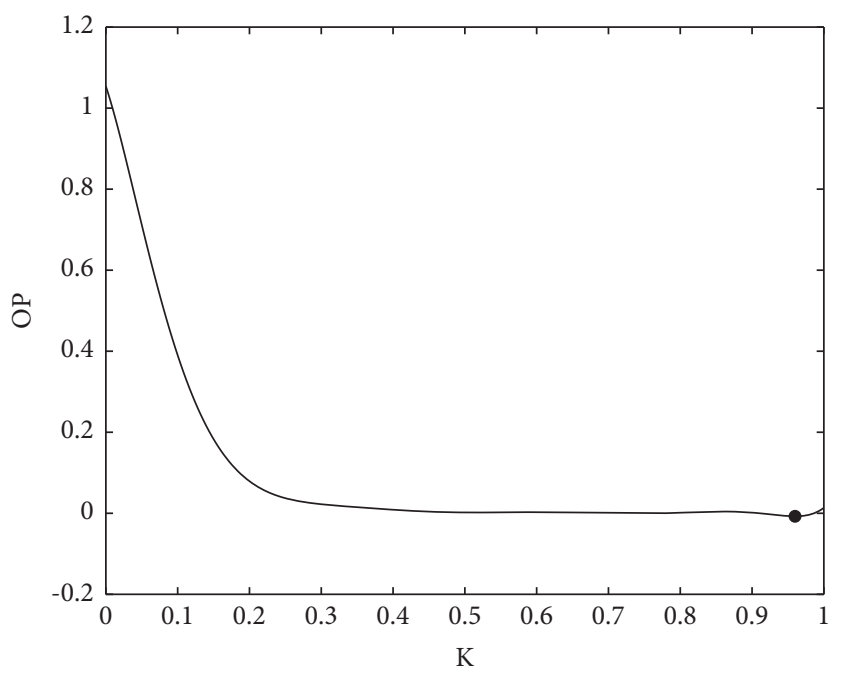

Figure 7: Optimum $K$ of FA.

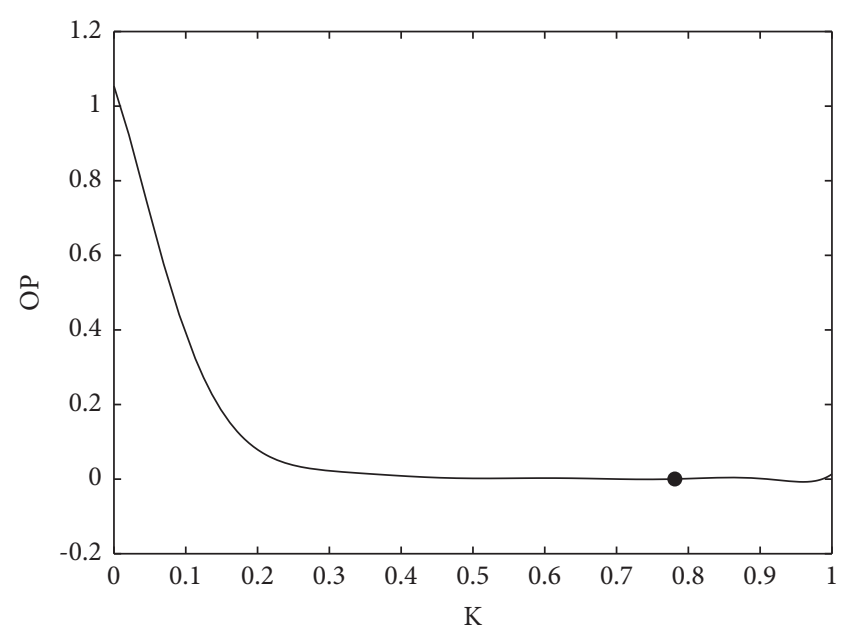

Figure 8: Optimum $K$ of GS.

TABLE 4: Simulation parameters for the 4 methods.

\begin{tabular}{lc}
\hline Algorithm & Simulation parameters \\
\hline GWO & psize $=50$, ter $=1000$ \\
GS & $a=0, b=1, \varepsilon=0.2$ \\
GA & psize $=50$, ter $=1000$ \\
FA & psize $=50, \alpha=0.5, \beta=0.2, \gamma=1$, ter $=1000$
\end{tabular}

\section{Conclusions}

In this paper, the power allocation optimization of mobile multiuser networks was investigated. Based on the GWO method, we proposed a power allocation optimization algorithm. The simulation results showed that compared with GS, GA, and FA algorithms, GWO algorithm can obtain better OP performance results. Compared with Nakagami and Rayleigh channel models, the 2-Rayleigh model has an increase of $81.2 \%$ and $66.6 \%$ in OP values, respectively.

In future studies, we will consider using artificial intelligence to obtain the optimal $K$ value.

\section{Data Availability}

The data used to support the findings of this study are available from the corresponding authors upon reasonable request and with permission of funders.

\section{Conflicts of Interest}

The authors declare that they have no conflicts of interest.

\section{Acknowledgments}

This study was supported by the Research Project of Teaching Reform in Higher Education Institutions in Jiangxi Province, China (JXJG-20-15-9). 


\section{References}

[1] S. Mumtaz, A. Al-Dulaimi, H. Gacanin, and A. Bo, "Block chain and big data-enabled intelligent vehicular communication," IEEE Transactions on Intelligent Transportation Systems, vol. 22, no. 7, pp. 3904-3906, Jul. 2021.

[2] H. Wang, X. Li, R. H. Jhaveri et al., "Sparse Bayesian Learning based channel estimation in FBMC/OQAM industrial IoT networks," Computer Communications, vol. 176, no. 8, pp. 40-45, Aug. 2021.

[3] L. W. Xu, X. P. Zhou, M. A. Khan, X. W. Li, V. G. Menon, and $\mathrm{X}$. $\mathrm{Yu}$, "Communication quality prediction for internet of vehicle (IoV) networks: an elman approach," IEEE Transactions on Intelligent Transportation Systems, 2021.

[4] F. Q. Wen, J. P. Shi, and Z. Zhang, "Generalized spatial smoothing in bistatic EMVS-MIMO radar," Signal Processing, vol. 193, Article ID 108406, 2022.

[5] J. Shi, Z. Yang, and Y. Liu, "On parameter identifiability of diversity-smoothing-based MIMO radar," IEEE Transactions on Aerospace and Electronic Systems, 2021.

[6] D. Kong, X. Zheng, Y. Zhang, and T. Jiang, "Frame repetition: a solution to imaginary interference cancellation in FBMC/ OQAM systems," IEEE Transactions on Signal Processing, vol. 68, no. 2, pp. 1259-1273, 2020.

[7] S. Wang and X. Jiang, "Three-dimensional cooperative positioning in vehicular ad-hoc networks," IEEE Transactions on Intelligent Transportation Systems, vol. 22, no. 2, pp. 937-950, 2021.

[8] H. Lei, Z. Yang, K.-H. Park et al., "Secrecy outage analysis for cooperative NOMA systems with relay selection schemes," IEEE Transactions on Communications, vol. 67, no. 9, pp. 6282-6298, 2019.

[9] A. H. A. El-Malek, A. M. Salhab, and S. A. Zummo, "New bandwidth efficient relaying schemes in cooperative cognitive two-way relay networks with physical layer security," IEEE Transactions on Vehicular Technology, vol. 66, no. 6, pp. 5372-5386, 2017.

[10] L. Xu, H. Wang, and T. A. Gulliver, "Outage probability performance analysis and prediction for mobile IoV networks based on ICS-BP neural network," IEEE Internet of Things Journal, vol. 8, no. 5, pp. 3524-3533, 2021.

[11] P. He and M. Dong, "Energy-efficient power allocation maximization with mixed group sum power bound and QoS constraints," IEEE Transactions on Communications, vol. 67, no. 10, pp. 7139-7151, 2019.

[12] Y. Xu, Z. Gao, Z. Wang, C. Huang, Z. Yang, and C. Yuen, "RIS-enhanced WPCNs: joint radio resource allocation and passive beamforming optimization," IEEE Transactions on Vehicular Technology, vol. 70, no. 8, pp. 7980-7991, 2021.

[13] B. Li, L.-L. Yang, R. G. Maunder, and S. Sun, "Resource allocation in millimeter-wave multicarrier-division duplex systems with hybrid beamforming," IEEE Transactions on Vehicular Technology, vol. 70, no. 8, pp. 7921-7935, 2021.

[14] M. D. L. Filomeno, M. L. R. de Campos, H. V. Poor, and M. V. Ribeiro, "Hybrid power line/wireless systems: an optimal power allocation perspective," IEEE Transactions on Wireless Communications, vol. 19, no. 10, pp. 6289-6300, 2020.

[15] M. Y. Arafat and S. Moh, "Localization and clustering based on swarm intelligence in UAV networks for emergency communications," IEEE Internet of Things Journal, vol. 6, no. 5, pp. 8958-8976, 2019.
[16] X. Wu, Y. Yin, L. Xu, X. Wu, F. Meng, and R. Zhen, "MultiUAV task allocation based on improved genetic algorithm," IEEE Access, vol. 9, Article ID 100369, 2021.

[17] Q. T Vien, T. A Le, X. S Yang, and T. Q Duong, "Enhancing security of MME handover via fractional programming and firefly algorithm," IEEE Transactions on Communications, vol. 67, no. 3, pp. 6206-6220, 2019.

[18] E. Cuevas, L. Enríquez, D. Zaldívar, and M. Pérez-Cisneros, "A selection method for evolutionary algorithms based on the golden section," Expert Systems with Applications, vol. 106, no. 9, pp. 183-196, 2018.

[19] G. K. Karagiannidis, N. C. Sagias, and P. T. Mathiopoulos, "\$N $\{$ last $\$$ Nakagami: a novel stochastic model for cascaded fading channels," IEEE Transactions on Communications, vol. 55, no. 8, pp. 1453-1458, 2007.

[20] L. Xu, X. Yu, and T. A. Gulliver, "Intelligent outage probability prediction for mobile IoT networks based on an IGWOElman neural network," IEEE Transactions on Vehicular Technology, vol. 70, no. 2, pp. 1365-1375, 2021.

[21] E. Emary, H. M. Zawbaa, and C. Grosan, "Experienced gray wolf optimization through reinforcement learning and neural networks," IEEE Transactions on Neural Networks and Learning Systems, vol. 29, no. 3, pp. 681-694, 2018.

[22] X. Zhang, Q. Kang, J. Cheng, and X. Wang, "A novel hybrid algorithm based on biogeography-based optimization and grey wolf optimizer," Applied Soft Computing, vol. 67, no. 6, pp. 197-214, 2018. 